\title{
Flow Cytometry Monitors Xylitol Metabolism of Streptococcus mitis
}

\section{Prabani Dissanayake ${ }^{3}$, Nardhy Gomez-Lopez ${ }^{1}$, Gail Czarnecki ${ }^{2}$ and Sunil Palchaudhuri ${ }^{3 *}$}

${ }^{1}$ Obstetrics and Gynecology \& Perinatology Research Branch, School of Medicine, Wayne State University, Detroit, Michigan 48201, USA

${ }^{2}$ Department of Orthodontics and Pediatric Dentistry, School of Dentistry, University of Michigan, Ann Arbor, MI, 48109, USA

${ }^{3}$ Department of Immunology and Microbiology, University of Michigan, Ann Arbor, MI, 48109, USA

\begin{abstract}
In this work we are presenting our novel adaptation of flow cytometry for the determination of change of diplococcic Gram-positive Streptococcus mitis population heterogeneity by their xylitol metabolism. The inherent population heterogeneity of the bacteria due to their growth in-chains of varying lengths was grouped into three different groups by flow cytometric analysis, designated as gates P1, P2 and P3. Gate P1 consists of the bacterial subpopulation with minimum cell wall thickness and therefore the least side scattering, while gate P3 consists of the subpopulation with the most side scattering (corresponding to the thick bacterial cell wall). According to our results gate P1 contains bacteria in long-chains, while P3 contains individual bacteria. When these diplococcic bacteria were grown in the presence of $2 \%$ xylitol more homogeneous population was seen as P1 gate was populated with approximately $80 \%$ of the total population. These results suggest that once xylitol is metabolized by these bacteria, they stabilize in long chains. These 'xylitol stabilized' long chains arose apparently by incomplete bacterial separation, contains individual bacteria with reduced cell wall thickness, resulted less side scattering. The optimum population homogeneity was achieved when these bacteria were grown in $2 \%$ xylitol and $300 \mathrm{ppm}$ fluoride containing medium, as measured by the bacterial population percentage in gate P1. Since these bacteria in long chains are known to be in a latent phase, our findings demonstrate that if appropriately developed xylitol has potential to use as an alternative antimicrobial for life threatening diseases.
\end{abstract}

Keywords: Xylitol; Flow cytometry; Population heterogeneity; Diplococcic S. mitis

\section{Introduction}

Low calorie low cost xylitol, a natural five carbon sugar-alcohol, is known to be affecting the genetic continuity of Streptococcus pneumoniae (causing pneumonia) and important members of the viridans group streptococci, including $S$. mutans (involved in dental caries), S. mitis (associated with bacteremia, meningitis, periodontal disease and pneumonia) [1-7]. These pathogens have similar diplococcic morphology and it is known that Streptococcus mitis bacterial species has several similar physiological characteristics common to $S$. pneumoniae including the ability to partially metabolize xylitol to produce a toxic xylitol intermediate, probably xylitol-5phosphate [8-14]. Our Raman spectroscopic studies have recently confirmed the formation of this xylitol intermediate by the metabolism of xylitol in both Gram-positive and Gram-negative bacteria, provided they contained the xylitol operons [15]. Moreover, we have shown that xylitol exposure interferes with the ability of these bacteria to retain the crystal violet, a dye essential in Gram staining technique [15]. A thinning of bacterial cell wall thickness may have occurred during their growth in xylitol, resulting in this reduced retention of crystal violet molecules. In addition, other studies on the ultra-structures of xylitol exposed pneumococci, have shown diffused cell wall structure, and thinly dispersed polysaccharide capsule [16]. Besides the Gram staining color difference, we have shown that diplococcic individuals are more prone to exist in long chains once xylitol is uptaken and metabolized by them [15]. We believe that xylitol metabolism results incomplete cleavage of the cross linkages of peptidoglycan layers during bacterial cell division. As a result, formation of diplococcic individuals in longchains becomes more feasible [17]. However, it has been reported that diplococcic individuals or diplococcic individuals in shortchains are necessary for virulence of pneumococci $[17,18]$. Since these bacteria display morphological heterogeneity with respect to chain length, they maintain an avirulent commensal status (long-chains) as well as pathogenic status (short-chains) [18,19]. In combination of these observations, and our previous findings we postulate that morphological heterogeneity with regard to chain length of these Gram-positive bacteria can be reduced by their growth in xylitol (2\% or higher). As a result these bacteria may exist in commensal status with 'xylitol stabilized' long chains. In this work, we examined the change of the total bacterial population heterogeneity upon xylitol metabolism by adapting flow cytometry and a well-defined overall physiological state was revealed in terms of their heterogeneity. This technique is being used as a powerful tool to characterize mechanisms of both bacterial and viral pathogenesis due to its simultaneous analysis capability of several important parameters together with specificity for both qualitative and quantitative analysis [20-25]. Since technique(s) with high accuracy and fast scanning ability is a must to confirm the xylitol metabolism by the entire population of pathogens, our adaptation of flow cytometry is well suited for such analysis.

Here we present our novel adaptation of flow cytometry as a simple, accurate and fast technique to monitor in vivo changes of population heterogeneity of Streptococcus mitis apparently induced by the metabolism of xylitol. These studies are important when determining the potential application of xylitol as an alternative preventive antimicrobial therapy for these life-threatening bacterial infections.

${ }^{*}$ Corresponding author: Sunil Palchaudhuri, Professor of Immunology and Microbiology, Wayne State University School of Medicine, Detroit, MI.48201, USA Tel: 313-577-1313; E-mail: spalchau@med.wayne.edu

Received November 13, 2013; Accepted December 11, 2013; Published December 18, 2013

Citation: Dissanayake P, Gomez-Lopez N, Czarnecki G, Palchaudhuri S (2014) Flow Cytometry Monitors Xylitol Metabolism of Streptococcus mitis. J Mol Genet Med 7: 095 doi: 10.4172/1747-0862.1000095

Copyright: (C) 2014 Gomez-Lopez N, et al. This is an open-access article distributed under the terms of the Creative Commons Attribution License, which permits unrestricted use, distribution, and reproduction in any medium, provided the original author and source are credited 


\section{Methods and Materials}

\section{Bacterial strains and experimental conditions}

A Gram-positive, catalase negative, non-hemolytic $(\gamma)$, and bileesculin negative 'pure' cultures of Streptococcus mitis (ATCC 6249) were grown overnight (10 to $14 \mathrm{~h}$ ) in brain heart infusion (BHI containing dextrose (5 g/l), DIFCO, Becton Dickinson) $37^{\circ} \mathrm{C}$. The overnight cultures of Streptococcus mitis were diluted 100 fold in fresh BHI containing medium as one experimental set, and the other set of experiments were performed by diluting the overnight culture by 10 , 000 fold. Xylitol stock solution $(20 \%)$, pre-warmed to $37^{\circ} \mathrm{C}$, was added to varying concentrations, 0 to $6 \%$ without diluting the growth media. A well characterized strain of E. coli $\mathrm{K}-12$ was used as a Gram negative internal control for thin cell wall, as well as absence of xylitol loci [15].

Bacterial growth was monitored by counting the colony forming units on a solid BHI agar media after appropriate dilutions as well as by measuring optical density at $580 \mathrm{~nm}$.

\section{Flow cytometric analysis}

In each experiment bacterial cells were harvested by centrifugation after overnight $(14 \mathrm{~h})$ incubation at $37^{\circ} \mathrm{C}$. The pellet was fixed with $1 \%$ freshly prepared formaldehyde solution for $1 \mathrm{~h}$ at ambient temperature and washed twice in phosphate buffered saline (PBS). Bacterial cells were re-suspended in PBS and adjusted to an $\mathrm{OD}_{580 \mathrm{~nm}}$ of approximately 0.8 .

Bacteria were then analyzed by flow cytometry (LSRFortessa, Becton Dickinson), acquiring 10,000 events within the characteristic flame-shaped region in the Forward Scatter Count (FSC)/ Side Scatter Count (SSC) dot plot, which excludes debris and large cells. FCS parameter measures the light scattered less than 10 degrees as a cell passes through the laser beam. The FSC measurement is related to cell size. SSC parameter measures the light scattered at a 90 degree angle as a cell passes through the laser beam. This measurement is related to the internal granularity or cell surface roughness. We gated 3 subpopulations of bacteria according SSC (Side Scatter Count) /FCS (Forward Scatter Count): P1 - 50K/100K; P2 - 150K/100K and P3 $250 \mathrm{~K} / 100 \mathrm{~K}$. For all our analyzed samples we kept this gate selection constant for easy and accurate comparison.

\section{Xylitol-Flouride experiments}

Varying concentrations of xylitol (0 to 6\%) and fluoride (0 to 300 ppm NaF (Bio labs) were added simultaneously for each culture (the overnight cultures of Streptococcus mitis diluted either 100 fold or 10, 000 fold) and grown overnight at $37^{\circ} \mathrm{C}$.

Gram-stain preparations of $S$. mitis were made after growing similary in BHI medium. These experiments were repeated several times using the standard Gram-staining technique and optical microscopy (final magnification 1000-fold).

For each condition in this study at least 3 replicates were performed and Students t-test was performed to calculated std.error.

\section{Results}

\section{Population heterogenity of diplococcic Streptococcus mitis based on cell wall thickness}

When Streptococcus mitis cultures were grown overnight at $37^{\circ} \mathrm{C}$ in the absence of xylitol (control sample) heterogeneity of population with respect to cell wall thickness was seen (Figure 1A). In Figure 1A, P1 gate (percentage of bacterial population due to the least side

scattering), related to 33.3 percent of bacteria, while $\mathrm{P} 2$ gate (percentage of bacterial population due to the medium side scattering), and $\mathrm{P} 3$ gate (population due to the most side scattering) represent 58.0, and 7.7 percent respectively.

To facilitate the uptake of xylitol by breakage of bacterial chains (growth phase metabolism) we changed the bacterial number by diluting overnight cultures of Streptococcus mitis by 100 fold in one set, and by 10,000 in the other set before grown in the presence of xylitol. When overnight cultures of Streptococcus mitis were diluted by 100 fold and were grown in the presence of xylitol (2 to 6\%) differences of heterogeneity were observed, compared to that of the control $(S$. mitis without xylitol) (Table 1A). Moreover, when overnight cultures of Streptococcus mitis were 10,000 fold diluted and grown in the BHI medium containing varying concentrations of xylitol (up to 6\%) further reductions of heterogeneity were seen; P1 population percentage have been further increased, while $\mathrm{P} 2$ and $\mathrm{P} 3$ populations have been decreased (Figure 1B and Table 1B). In contrast, the population percentages observed when overnight cultures of Streptococcus mitis were 100 fold diluted and grown without xylitol were not different to the population percentages observed when overnight cultures were 10,000 fold diluted and grown without xylitol at 95\% confidence interval (Student $\mathrm{t}$ test) (Table 1A and Table 1B).

Interestingly, when overnight cultures of Streptococcus mitis were 10,000 fold diluted and grown in the presence of varying concentrations of xylitol, the maximum P1 population percentage was achieved for 2\% xylitol treated Streptococcus mitis and P1 population percentages observed for $4 \%$ and 6\% xylitol treated Streptococcus mitis were not different to that of 2\% xylitol treated Streptococcus mitis at 95\% confidence interval (Student $t$ test) (Table 1B).

When similar studies were performed using E. coli $\mathrm{K}-12$ as an internal control for homogeneous population, their total population was seen in gate P1 (Figure 2A). Furthermore, the bacterial population distribution was not significantly changed for $E$. coli K-12 when similar xylitol experiments were carried out. Figure 2B shows the unaffected distribution of $E$. coli $\mathrm{K}-12$ population in the presence of xylitol (2\%).

\section{Efficacy of xylitol in combination with fluoride}

The population heterogeneity observed for any combination of xylitol (up to $6 \%$ ) and fluoride (10 to $200 \mathrm{ppm}$ ) treated samples were not different to xylitol (up to 6\%) treated samples. However, when higher concentrations of fluoride were used $(300 \mathrm{ppm})$ in all combinations

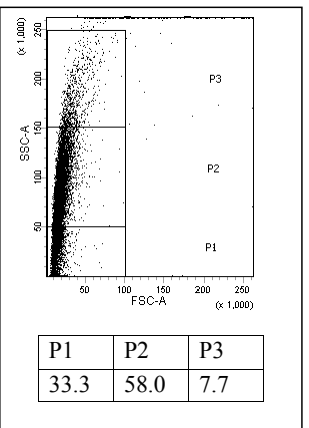

Figure 1: Dot-plots showing the heterogeneity of populations of Streptococcus mitis grown for $14 \mathrm{~h},(\mathrm{~A})$ without xylitol, (B) grown similarly in medium containing xylitol $(2 \%)$. 
with 2 to $6 \%$ xylitol, P1 percentages increased significantly. Interestingly, when overnight cultures of Streptococcus mitis were diluted to 10,000 fold and grown overnight in the presence of xylitol, 0 to $6 \%$ with 300 ppm fluoride, the percentage of $\mathrm{P} 1$ population reached the maximum and gets plateaued at $2 \%$ xylitol condition. Both $\mathrm{P} 2$, and $\mathrm{P} 3$ population percentages were further decreased with the combined effect of fluoride and xylitol. Data obtained for $2 \%$ xylitol and $300 \mathrm{ppm}$ fluoride combination is shown in Figure 3 as representative data.

\section{Viabilty analysis of diplococcic Streptococcus mitis grown in Xylitol}

In order to confirm that these Streptococcus mitis are all viable after exposing to xylitol (upto 6\%), we spread them on BHI-agar medium, and counted the colony forming units after overnight incubation at $37^{\circ} \mathrm{C}$. We have observed comparable bacterial colony forming units.

\section{Gram staining and optical microscopy}

The Gram-stain preparations for the control (grown without xylitol) revealed all purple diplococci together with a small percentage of smaller spherical ones, poorly stained (indicated by arrows in Figure 4).

\section{Discussion}

The Gram-positive Streptococcus bacteria grow in chains of variable lengths [18]. The heterogeneous population thus created with respect bacterial morphology and size consists of diplococcic individuals,

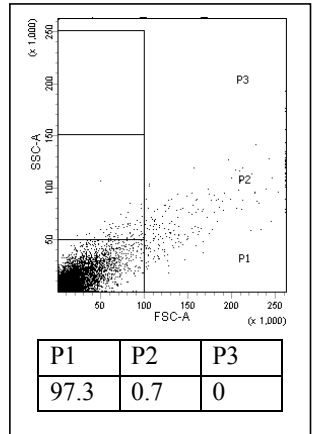

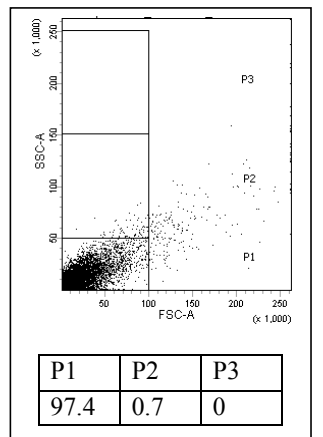

Figure 2: Dot-plots showing the homogeneity of populations of E.coli K-12 (A) grown for $14 \mathrm{~h}$ and (B) similarly grown with $2 \%$ xylitol.

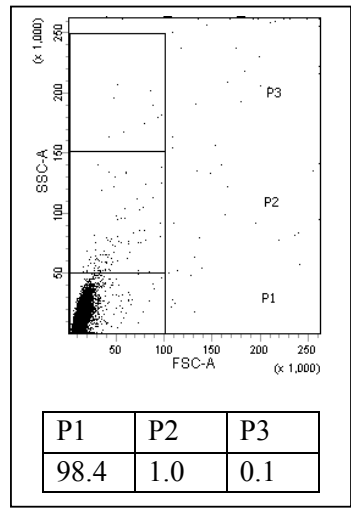

Figure 3: Dot-plots showing the heterogeneity of Streptococcus mitis when overnight cultures were diluted to 10,000 fold and grown for $14 \mathrm{~h}$ in the presence of $2 \%$ xylitol and 300 ppm fluoride.

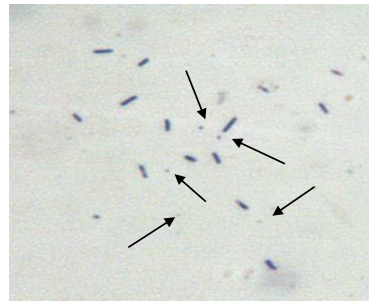

Figure 4: Overnight culture of S. mitis (10,000 diluted in BHI (Control)). Arrows show the presence of small spherical population, partially lacking cell walls.

diplococcic individuals in short-chain forms, and diplococcic in longchain forms. The chain formation of these bacteria results due to the incomplete cleavage of the peptidoglycan layers during bacterial cell division [17]. In the past, this bacterial heterogeneity arises due to bacterial cell division has been interpreted in terms of colony morphologies, such as rough (R) colonies and smooth (S) colonies [26]. However, many confusion and difficulties were arisen due to this classification based on the colony morphologies, and it has been reported later that these colony morphologies are not representing genotype differences, but merely the phenotypic differences resulted in cellular separation after cell division $[27,28]$. Moreover, due to the fragile nature of these bacterial chains, during the course of dilution techniques and spreading procedures these chains may get dissociated randomly leading to erroneous observations. Therefore, it is apparent that if these heterogeneities are characterized with respect to bacterial cellular attributes, the existing ambiguities resulted due to colonial classification with respect to their pathogenecity can be eliminated.

The present study shows our adaptation of flow cytometry as a fast and accurate tool in determining total bacterial population heterogeneity, with respect to their cell wall thickness. The inherent heterogeneity we observe for Gram -positive control samples of S. mitis in the absence of xylitol represents their growth-pattern consists of diplococcic individuals and chains of diplococcic individuals in varying length [18]. As shown in Figure 1A, inherent bacterial population heterogeneity has been gated with respect to side scattering count (SSC), which is related to bacterial cell wall thickness. We hypothesized that bacteria in chains are responsible for the least SSC, mostly the P1 gate, due to the thinly dispersed bacterial cell wall resulted by the incomplete cleavage of the peptidoglycan layers during bacterial cell division. In contrast individual diplococcic bacteria can give rise to higher SSC value as they have relatively thick bacterial cell wall. Therefore according to our classification P3 gate consist of individual diplococcic bacteria, while P2 gate has individual bacteria in short chains.

In contrast to the heterogeneity we observed for Streptococcus mitis, a homogeneous population of $E$. coli $K-12$ was observed (Figure 2A), as almost total bacteria were populated in gate P1. Since E. coli $\mathrm{K}-12$ is not growing in chains of varying lengths, comparable SSC values were obtained for the total population due to the presence of a one kind thin cell wall thickness.

Interestingly, in the presence of xylitol (up to 6\%), more homogeneous Streptococcus mitis populations were resulted as appeared in population of P1 gate (Figure 1B). With our previous publication it confirms that due to the stable presence of xylitol derivative (as resulted by the xylitol metabolism) these diplococcic bacteria are more prone to exist as chains [15], hence P1 population increased. Moreover, the reduced SSC data in the presence of xylitol supports previously observed thinning of cell wall thickness of pnemococci due to the exposure of xylitol [16]. 
Based on the similaritities of the heterogeneties we have seen for the control (the Streptococcus mitis grown without xylitol) it is obvious that initial dilution factor (either 100 dilution of the overnight culture before exposing to xylitol, or 10,000 fold initial dilution overnight culture before exposing to xylitol) is not responsible for the observed heterogeneity change seen in xylitol exposed Streptococcus mitis. It seems like when overnight cultures were 100 fold diluted there may be a percentage of bacterial population still in chains, and are not capable of uptaking xylitol. Another possibility is when overnight cultures were 100 fold diluted there are more bacteria to begin with, therefore xylitol can be a limiting factor. Therefore, if we increased the concentration of xylitol from 1 to $6 \%$ by keeping the dilution factor a constant (100 fold), the chance for bacteria to utilize xyliol should be increased, resulting higher percentage for P1 population. Results shown in Table 1A, support this phenomenon. Further it was clear from Table 1B data that upon higher initial dilution $(10,000)$, $\mathrm{P} 1$ population reaches maximum when grown in the presence of optimum concentration of xylitol (2\%). This supports that at higher initial dilution $(10,000)$, Streptococcus mitis in-chains have been converted to individual diplococcic which can actively metabolize xylitol, and xylitol is not becoming the limiting factor. However, based on our xylitol experimental results, even with 10,000 dilutions the maximum P1 population observed was about $90 \%$, suggesting the presence of non-growing Streptococcus mitis probably with chains. Since it has been reported that chaining of streptococci can be reduced by the action of fluoride due to the loss of glucanbinding lectin activity [29], in order to increase the efficacy of xylitol we have combined fluoride with xylitol. Fluoride concentration up to $300 \mathrm{ppm}$ were not effective enough to show significant changes of the heterogeneity as measured by the flow cytometry. By the results of our xylitol-fluoride experiments, it seems like the remaining individual diplococci in-chains (after 10,000 fold dilution of the overnight culture) have been dissociated into individual diplococci which can efficiently metabolize xylitol. Thus, the percentage of P1 due to 'xylitol stabilized' chains increased significantly as seen in Figure 3. In our experiments, the condition with the minimum amount of xylitol and minimum amount of fluoride in a bacterial cultures which gave the highest $\mathrm{P} 1$ population was defined as the optimum combined condition As shown in Figure 3 , the optimum combined effect was seen when overnight cultures of Streptococcus mitis were diluted to 10,000 fold and grown overnight in the presence of $2 \%$ xylitol, and $300 \mathrm{ppm}$ fluoride. However, if 100 fold initial dilution was used in order to obtain the optimum combined condition, $6 \%$ xylitol in combination with $300 \mathrm{ppm}$ fluoride had to be used. Moreover, if xylitol concentration was reduced to $2 \%$, the fluoride concentration had to be increased to $600 \mathrm{ppm}$ to see the same effect (results not shown). These results suggest that not only the xylitol, and fluoride concentration, the initial dilution of the overnight culture is also playing an important role in determining their heterogeneity with respect to chain lengths of Streptococcus mitis.

The confirmative determination of the changes of morphological heterogeneity of bacteria due to the effect of antimicrobial drugs, in our case xylitol, the results obtained only from the colonial appearance are not sufficient enough. The reasons include, the use of only a very small fraction of the actual bacterial culture as a representative sample after several serial dilution steps (these experimental steps may dissociate bacterial chains into diplococcic individuals), and relative slowness as we have to grow the microorganisms in appropriate growth media. Therefore, it is evident from our studies that flow cytometry can be adapted as an efficient, and accurate tool for monitoring the changes of total bacterial population heterogeneity when determining the applicability of xylitol and/ fluoride as an alternative antimicrobial therapy against pathogenic bacteria.

\section{Acknowledgement}

We would like to thank Dr. A. Palchaudhuri for the financial support.

\section{References}

1. Honkala E, Honkala S, Shyama M, Al-Mutawa SA (2006) Field trial on caries prevention with xylitol candies among disabled school students. Caries Res 40: $508-513$

2. Kontiokari T, Uhari M, Koskela M (1995) Effect of xylitol on growth of nasopharyngeal bacteria in vitro. Antimicrob Agents Chemother 39: 1820-1823.

3. Mäkinen KK, Alanen $P$, Isokangas $P$, Isotupa K, Söderling E, et al. (2008) Thirty-nine-month xylitol chewing-gum programme in initially 8-year-old school children: a feasibility study focusing on mutans streptococci and lactobacilli. Int Dent J 58: 41-50.

4. Söderling EM, Ekman TC, Taipale TJ (2008) Growth inhibition of Streptococcus mutans with low xylitol concentrations. Curr Microbiol 56: 382-385.

5. Tapiainen T (2002) Microbiological effects and clinical uses of xylitol in preventing acute otitis media. PhD Thesis, University of Oulu, Oulu, Finland.

6. Trahan L (1995) Xylitol: a review of its action on mutans streptococci and dental plaque--its clinical significance. Int Dent J 45: 77-92.

7. Uhari M, Tapiainen T, Kontiokari T (2000) Xylitol in preventing acute otitis media. Vaccine 19 Suppl 1: S144-147.

8. Gladwin M, Trattler B (2011) Clinical Microbiology made ridiculously simple 5th ed: Gram-positive Bacteria, Streptococci. MedMaster, Inc. Miami, FL.

9. Patterson MJ (1996) Medical Microbiology (4th edn) Streptococcus Galveston, TX.

10. Ikryannikova LN, Filimonova AV, Malakhova MV, Savinova T, Filimonova $O$, et al. (2013) Discrimination between Streptococcus pneumoniae and Streptococcus mitis based on sorting of their MALDI mass spectra. Clin Microbiol Infect 19: 1066-1071.

11. Madhour A, Maurer P, Hakenbeck R (2011) Cell surface proteins in $S$. pneumoniae, S. mitis and S. oralis. Iran J Microbiol 3: 58-67.

12. Martín-Galiano AJ, Balsalobre L, Fenoll A, de la Campa AG (2003) Genetic characterization of optochin-susceptible viridans group streptococci. Antimicrob Agents Chemother 47: 3187-3194.

13. Janoir C, Podglajen I, Kitzis MD, Poyart C, Gutmann L (1999) In vitro exchange of fluoroquinolone resistance determinants between Streptococcus pneumoniae and viridans streptococci and genomic organization of the parEparC region in S. mitis. J Infect Dis. 180: 555-558.

14. Balsalobre L, Ferrándiz MJ, Liñares J, Tubau F, de la Campa AG (2003) Viridans group streptococci are donors in horizontal transfer of topoisomerase IV genes to Streptococcus pneumoniae. Antimicrob Agents Chemother 47: 2072-2081.

15. Palchaudhuri S, Rehse SJ, Hamasha K, Syed T, Kurtovic E, et al. (2011) Raman spectroscopy of xylitol uptake and metabolism in Gram-positive and Gram-negative bacteria. Appl Environ Microbiol 77: 131-137.

16. Tapiainen $T$, Sormunen $R$, Kaijalainen $T$, Kontiokari $T$, Ikäheimo I, et al (2004) Ultrastructure of Streptococcus pneumoniae after exposure to xylitol. J Antimicrob Chemother 54: 225-228.

17. Dalia AB, Weiser JN (2011) Minimization of bacterial size allows for complement evasion and is overcome by the agglutinating effect of antibody. Cell Hos Microbe 10: 486-496.

18. Rodriguez JL, Dalia AB, Weiser JN (2012) Increased chain length promotes pneumococcal adherence and colonization. Infect Immun 80: 3454-3459.

19. Weiser JN (2010) The pneumococcus: why a commensal misbehaves. J Mol Med (Berl) 88: 97-102.

20. Steen HB, Boye E, Skarstad K, Bloom B, Godal T, et al. (1982) Applications of flow cytometry on bacteria: cell cycle kinetics, drug effects, and quantitation of antibody binding. Cytometry 2: 249-257.

21. Steen HB, Boye E (1981) Escherichia coli growth studied by dual-parameter flow cytophotometry. J Bacteriol 145: 1091-1094. 
Citation: Dissanayake P, Gomez-Lopez N, Czarnecki G, Palchaudhuri S (2014) Flow Cytometry Monitors Xylitol Metabolism of Streptococcus mitis. J Mol Genet Med 7: 095 doi: 10.4172/1747-0862.1000095

Page 5 of 5

22. Allman R, Hann AC, Manchee R, Lloyd D (1992) Characterization of bacteria by multiparameter flow cytometry. J Appl Bacteriol 73: 438-444.

23. Pinder AC, Purdy PW, Poulter SA, Clark DC (1990) Validation of flow cytometry for rapid enumeration of bacterial concentrations in pure cultures. J App Bacteriol 69: 92-100.

24. Laffin J, Lehman JM (1994) Detection of intracellular virus and viral products. Methods Cell Biol 41: 543-557.

25. Lehman JM, Jacobberger JW (1990) Virus-cell interactions analyzed with flow cytometry. Flow cytometry and sorting. Wiley-Liss, Inc., New York, N.Y.
26. Austrian R (1953) Morphologic variation in pneumococcus. I. An analysis of the bases for morphologic variation in pneumococcus and description of a hitherto undefined morphologic variant. J Exp Med 98: 21-34.

27. Nutt MM (1927) The Method of Division of the Rough and Smooth Type of Colonies among Bacilli of the Salmonella Group. J Hyg (Lond) 26: 44-48.

28. Bisset, KA (1938) J Path and Bact 47: 223.

29. Cox SD, Lassiter MO, Miller BS, Doyle RJ (1999) A new mechanism of action of fluoride on streptococci. Biochim Biophys Acta 1428: 415-423. 